\title{
Effectiveness of Thymoquinone and Fluoridated Bioactive Glass/Nano-Oxide Contained Dentifrices on Abrasion and Dentine Tubules Occlusion: An Ex Vivo Study
}

\author{
Daud Anthoney ${ }^{1}$ Shahreen Zahid ${ }^{2,3}$ Hina Khalid ${ }^{4,5}$ \\ Aqif Anwar Chaudhry ${ }^{4}$ Abdul Samad Khan ${ }^{7}$

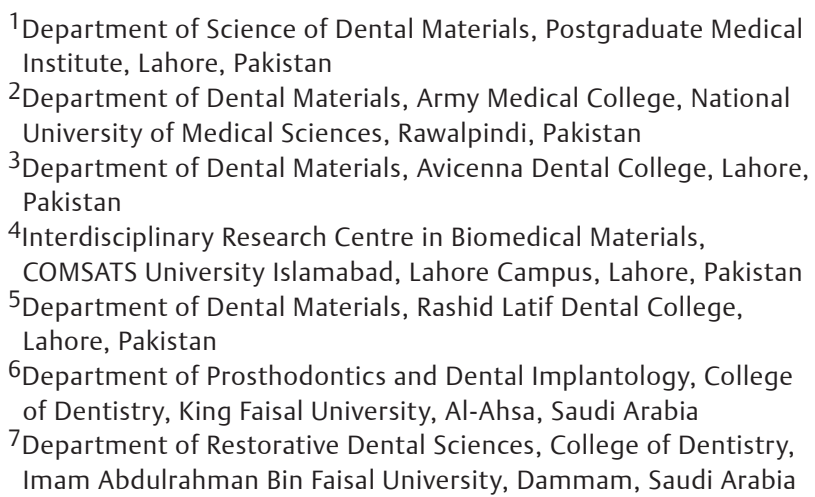

Eur J Dent 2020;14:45-54

Zohaib Khurshid $^{6}$ Asma Tufail Shah ${ }^{4}$

\begin{abstract}
Address for correspondence Abdul Samad Khan, BDS, MSc, PhD, MADM, Department of Restorative Dental Sciences, College of Dentistry, Imam Abdulrahman Bin Faisal University, Dammam 31441, Saudi Arabia (e-mail: akhan@iau.edu.sa).
\end{abstract}

Abstract

Keywords

- dentin sensitivity

- dentifrices

- abrasion

- bioactive glasses

- zinc oxide

- tooth remineralization
Objectives Dentin hypersensitivity (DH) is mainly due to the loss and replenishment of minerals from tooth structure, where the lost minerals can be rehabilitated with a biomimetic approach. The objectives were to determine the relative dentin abrasivity (RDA) of experimental (EXT) dentifrices and to determine the efficacy to occlude dentinal tubules.

Materials and Methods Experimental dentifrices contained nano-fluoridated bioactive glass (n-FBG: 1.5 wt.\% [EXT-A], 2.5 wt.\% [EXT-B], and 3.5 wt.\% [EXT-C]), nano-zinc oxide $(\mathrm{n}-\mathrm{ZnO})$, and thymoquinone as active agents. Bovine dentin blocks were subjected to brushing treatments as per groups, that is, distilled water; commercial dentifrice (control, CT); EXT toothpastes; and EXT-D without active agents. Samples were tested for three-dimensional (3D) abrasion analysis according to ISO-11609:2010 (International Organization for Standardization [ISO]). The roughness average (Ra), RDA, surface topography, and elemental compositions were investigated.

Statistical Analysis One-way analysis of variance (ANOVA) with post-hoc Tukey's and Tamhane's test was performed for characterizations using Statistical Package for the Social Sciences (SPSS) version 21. The result was considered significant with $p$-value $\leq 0.05$.

Results Comparisons of Ra differed significantly between all groups with $p<0.05$ except CT and EXT-A. The RDA values of EXT-A, EXT-B, and EXT-C were calculated as 74.04, 84.26, and 116.24 , respectively, which were well within the acceptable limit set by international standards. All n-FBG containing dentifrices demonstrated uniform occlusion of dentinal tubules; however, highly concentrated EXT dentifrices showed more occlusion.

Conclusions Acceptable range of RDA and superior occlusion of tubules by novel dentifrices suggest that it may be recommended for treating $\mathrm{DH}$.
DOI https://doi.org/

$10.1055 / \mathrm{s}-0040-1703418$

ISSN $1305-7456$.
License terms

(요 (1) $\Theta \circledast$ 


\section{Introduction}

Dentin hypersensitivity (DH) remains an oral health problem in adults on a global scale. Its prevalence is generally reported to be between 4 and 74\%, although it varies between different populations. ${ }^{1}$ Based on the "hydrodynamic theory" the main method to treat DH is by occlusion of dentinal tubules as it would decrease stimuli-evoked fluid shifts inside the dentinal tubules, thereby relieving symptoms of sensitivity. ${ }^{2,3}$

A desire to improve the quality of oral hygiene came up with many types of dentifrices, where a wide range of at-home desensitizing products including toothpastes, mouthwashes, and chewing gums are available to treat sensitized tooth structure. ${ }^{4,5}$ However, none have reliably demonstrated complete elimination of hypersensitivity because the occluding materials formed were not constituents of natural dentin and regular application of these materials is required. ${ }^{6,7}$

Recent research has highlighted the relevance and necessity of biomimetic materials such as bioactive glasses (BG) in treating DH. Dentifrices containing BG are effective in occluding open dentinal tubules and reducing sensitivity due to the formation of carbonated hydroxyapatite (HCA) ${ }^{8,9}$ Fluoride-containing bioactive glasses (F-BGs) have shown to be more beneficial due to the formation of fluorapatite/fluorohydroxyapatite (FA/F-HA), which is more acid-resistant than HCA. ${ }^{10,11}$

Nano-oxides such as nano-zinc oxide $(\mathrm{n}-\mathrm{ZnO})$ releases $\mathrm{Zn}^{2+}$ as therapeutically active ions for bactericidal and antigingivitis properties. ${ }^{12}$ Also, in recent times, considerable research has been done on using various types of plant extracts such as Azadirachta indica, Salvadore persica, and Nigella sativa in dentistry. ${ }^{13-15} \mathrm{~N}$. sativa contains thymoquinone having antibacterial and anti-inflammatory properties and could reduce dental caries and prevent periodontal and gingival diseases. ${ }^{16}$
The bioceramics have certain abrasivity and it needed to be evaluated to prevent the wear of hard tissues, that is, enamel and dentin. Toothpastes need a certain amount of abrasivity to perform the cleaning action. ${ }^{17}$ Abrasion is measured against a standard abrasive system to give relative enamel abrasivity (REA) or relative dentin abrasivity (RDA). The cleaning action of toothpastes is attributed to their abrasive values (REA and RDA), which should not exceed the limits set by the ISO standards..$^{18}$ Therefore, the present in vitro study was conducted to measure the RDA of newly formulated dentifrices. Moreover, to the authors' knowledge, no in vitro study has been conducted till the day, which not only measured the occluding potential of toothpaste formulations containing nano-fluoridated bioactive glass (n-FBG), n-ZnO, and therapeutic thymoquinone mentioned above, but also the abrasivity caused by these unique formulations.

\section{Materials and Methods}

\section{Preparation of Dentifrices}

Five dentifrices including control (CT) and experimental (EXT) were used in this study. To prepare the EXT dentifrices, initially, basic ingredients were mixed in increments and then $\mathrm{n}-\mathrm{ZnO}$ ( 3 wt.\%) and thymoquinone (3 wt.\%, purified black seed oil, Marhaba Laboratories Pvt. Ltd., Lahore, Pakistan) were added. Afterward, the n-FBG was mixed in various concentrations, that is, 1.5, 2.5, and $3.5 \% \mathrm{wt} / \mathrm{wt}$ and the EXT dentifrices were denoted on the basis of these concentrations as EXT-A, EXT-B, and EXT-C respectively. The $\mathrm{n}-\mathrm{FBG}$ and $\mathrm{n}-\mathrm{ZnO}$ were prepared by our group and were described previously. ${ }^{19,20}$ The composition of CT and the EXT dentifrices is given in - Table 1. Commercial dentifrice, that is, Colgate "Cavity Protection" (Colgate-Palmolive, Dublin, Ireland, Lot number:

Table 1 Composition of experimental and commercial dentifrices

\begin{tabular}{|c|c|c|}
\hline Groups & Composition & Active agents \\
\hline Experimental dentifrice (EXT-A) & \multirow{4}{*}{$\begin{array}{l}\mathrm{TiO}_{2}(1.5 \text { wt.\%) } \\
\text { Calcium carbonate (32 wt.\%) } \\
\text { Glycerol (27 wt.\%) } \\
\text { Sodium benzoate (1 wt.\%) } \\
\text { Methylcellulose (1.5 wt.\%) } \\
\text { Peppermint (Flavoring agent) (1 wt.\%) } \\
\text { Sodium lauryl sulfate (1 wt.\%) } \\
\text { Double distilled water (35\%) }\end{array}$} & $\begin{array}{l}\text { n-FBG (1.5 wt.\%) } \\
\text { n-ZnO (3 wt.\%) } \\
\text { Thymoquinone (black seed } \\
\text { oil, } 3 \text { wt.\%) }\end{array}$ \\
\hline Experimental dentifrice (EXT-B) & & $\begin{array}{l}\text { n-FBG ( } 2.5 \text { wt. } \%) \\
\text { n-ZnO (3 wt.\%) } \\
\text { Thymoquinone (black seed } \\
\text { oil, } 3 \text { wt.\%) }\end{array}$ \\
\hline Experimental dentifrice (EXT-C) & & $\begin{array}{l}\text { n-FBG (3.5 wt.\%) } \\
\text { n-ZnO (3 wt.\%) } \\
\text { Thymoquinone (black seed } \\
\text { oil, } 3 \text { wt.\%) }\end{array}$ \\
\hline Experimental dentifrice (EXT-D) & & No active ingredient \\
\hline $\begin{array}{l}\text { Control dentifrice }\left({ }^{\mathrm{a}} \mathrm{CT}\right) \text { Colgate-Palmolive, } \\
\text { Ireland }\end{array}$ & $\begin{array}{l}\text { Calcium carbonate, aqua, sorbitol, sodium lauryl sulfate, } \\
\text { aroma, cellulose gum, sodium bicarbonate, tetrasodium } \\
\text { pyrophosphate, benzyl alcohol, sodium saccharin, sodium } \\
\text { hydroxide, limonene }\end{array}$ & $\begin{array}{l}\text { Sodium monofluorophos- } \\
\text { phate, } 0.76 \%(0.15 \% \mathrm{w} / \mathrm{v} \\
\text { fluoride ion) } \\
\text { Dicalcium phosphate } \\
\text { dehydrate }\end{array}$ \\
\hline
\end{tabular}

Abbreviations: $\mathrm{n}$-FBG, nano-fluoridated bioactive glass; $\mathrm{n}$-ZnO, nano-zinc oxide.

${ }^{\mathrm{a}} \mathrm{CT}$, reference abrasive dentifrice $(\mathrm{RDA}=70)$. 
10131023) was used as reference dentifrice and it contained $0.76 \%$ sodium monofluorophosphate and $0.15 \% \mathrm{w} / \mathrm{v}$ fluoride ions.

\section{Dentin Specimen Preparation}

Sixty noncarious extracted bovine incisors were obtained under a protocol approved by the institutional ethical committee. Collected samples were scraped clean of soft tissue remnants and were disinfected with a 5\% sodium hypochlorite solution for 24 hours (ISO-11609:2010). Samples were stored in sterile $0.9 \%$ saline at $4^{\circ} \mathrm{C}$ for no longer than a month prior to use. Dentin blocks ( $4 \mathrm{~mm} \times 4 \mathrm{~mm} \times 2 \mathrm{~mm}$; length $\times$ width $\times$ height) were prepared by sectioning each tooth at the cementum-enamel junction (CEJ) using a water-cooled abrasive bur (MANI INC., Tochigi, Japan). Specimens were prepared from the buccal aspect of the teeth and buccal enamel was removed similarly to expose the mid-dentin. The back of all tooth samples was marked to recognize the buccal aspect of the tooth. The unmarked buccal surfaces were wet ground and polished gradually with 800- to 4,000-grit silicon carbide papers using a grinding-polishing machine (Struers Knuth Rotor 2, Denmark). These were then washed and sonicated in distilled water for 30 seconds to remove residual abrasives without affecting the structure. Samples were also etched with 37\% phosphoric acid (Meta Etchant; Meta Biomed Co. Ltd, Korea) for 15 seconds to expose the dentinal tubules and washed properly for 20 seconds and dried.

\section{Experimental Design}

Dentin specimens were randomly divided into six groups, where each contained 10 samples. Each tooth sample was coated with two layers of acid-resistant nail varnish (Max Factor International, Procter \& Gamble Ltd. Surrey, England) except for an exposed dentin window $\sim 2 \mathrm{~mm} \times 2 \mathrm{~mm}$ on the buccal surface. Following this, dentin specimens were positioned in a custom-made automated brushing machine with six brushing arms and a soft toothbrush (Oral B, Flat trim indicator, Oral B Procter and Gamble Ltd. Surrey, England) rested on each specimen with a load of $150 \mathrm{~g}$. Reference and test paste slurries were prepared by adding $25 \mathrm{~g}$ of each dentifrice to $40 \mathrm{~mL}$ (to develop one batch) of distilled water (1:1.6 w/v; ISO-11609:2010) and stirred using a magnetic stirrer (Corning Laboratory Stirrer/Hot Plate 6798-420 D, Mexico). The brush moved back and forth (one stroke) with a speed of $220 \mathrm{rpm}$ and 10,000 brushing cycles were applied on each specimen (ISO-11609:2010), which was estimated to be approximately 1.5 years of brushing simulation. ${ }^{21}$ Between each test, new brushes were mounted in the machine.

The groups corresponded to the following brushing treatments were: group 1 (negative $\mathrm{CT}$ ) treated with distilled water; group 2 (positive CT); group 3 (EXT-A); group 4 (EXT-B); group 5 (EXT-C); and group 6 (EXT-D). After brushing simulation of all the six groups, five samples out of each group were rinsed with distilled water only, whereas the other five samples were rinsed with distilled water followed by sonication (VGT-1860QTD, MTI Corporation, United States) for 30 seconds in an ultrasonic bath (Richmond, CA, United States).

\section{AFM Examination}

After treatment, the nail varnish was removed and surface roughness average (Ra) analysis of exposed and covered areas was performed with Atomic Force Microscope (SPM-9500J3, Shimadzu Corp, Kyoto,Japan) operating in the noncontact mode and atomic force microscopy (AFM) software (SPM-Offline, Shimadzu Corp, Kyoto, Japan). Typical three-dimensional (3D) images of abraded (after toothbrush simulation) and nonabraded dentin (polished surface) were reported in an area selection of $10.0 \mu \mathrm{m} \times 10.0 \mu \mathrm{m}$. The micrographs at six different areas $(2.0 \mu \mathrm{m} \times 2.0 \mu \mathrm{m}$ scan area $)$ were taken and the mean value was calculated for each specimen. RDA was calculated according to the formula mentioned in ISO-11609:2010. ${ }^{18}$

$$
\mathrm{RDA}_{\text {test }}=\frac{\mathrm{Ka}_{\text {test }} \times \mathrm{K \nu A}_{\text {ref }}}{\mathrm{Ra}_{\text {ref }}}
$$

where $\mathrm{RDA}_{\text {ref }}$ is 70 (known value).

\section{SEM-EDX Examination}

After the AFM examination, scanning electron microscopy (SEM) analysis was performed to assess the occlusion of dentinal tubules using a Scanning Electron Microscope (TESCAN VEGA-3 LMU, Brno, Czech Republic). Morphology of etched dentin was also observed. All samples were processed appropriately including dehydration and sputter coating with gold for electrical conductance in a high-vacuum sputter coating machine (SC7620 sputter coater; Quorum Technologies, Lewes, United Kingdom). Images were taken in the magnification range from $1,000 \times$ to $50,000 \times$ and were operated at an accelerated voltage of $8.0 \mathrm{kV}$. Energy dispersive X-ray (EDX) analysis (Oxford Instruments, Abingdon, United Kingdom) was also conducted for the identification of the abrasive elements.

\section{Tubule Occlusion Analysis}

Images (eight per group) depictive for every group were chosen for analysis. Three independent reviewers assessed the percentage of tubule occlusion from the SEM images. The reviewers were requested to report the percentage of tubule occlusion by assessing every tubule in the individual SEM image and report the mean percentage of occluded tubules in individual SEM micrographs based on the following criteria: No occlusion $=0 \%, 1 / 4=0-25 \%$ occluded, $2 / 4=25-50 \%$ occluded, $3 / 4=50-75 \%$ occluded, and $4 / 4=75-100 \%$ occluded. The percentage of the occluded dentinal tubules for each photomicrograph was calculated $\mathrm{as}^{22}$ :

$$
\text { Percentage of occluded tubules }=\frac{\text { No. } \text { of occluded tubules }}{\text { Total no. } \text { of tubules }} \times \mathbf{1 0 0}
$$

\section{Statistical Analysis}

Statistical Package for the Social Sciences (SPSS) version 21 (IBM SPSS Software, Atlanta, Georgia, United States) was used for statistical analysis. Mean and standard deviations of surface Ra of all groups were calculated by descriptive analysis. The difference between the groups was calculated by one-way analysis of variance (ANOVA). Post-hoc Tukey's and 

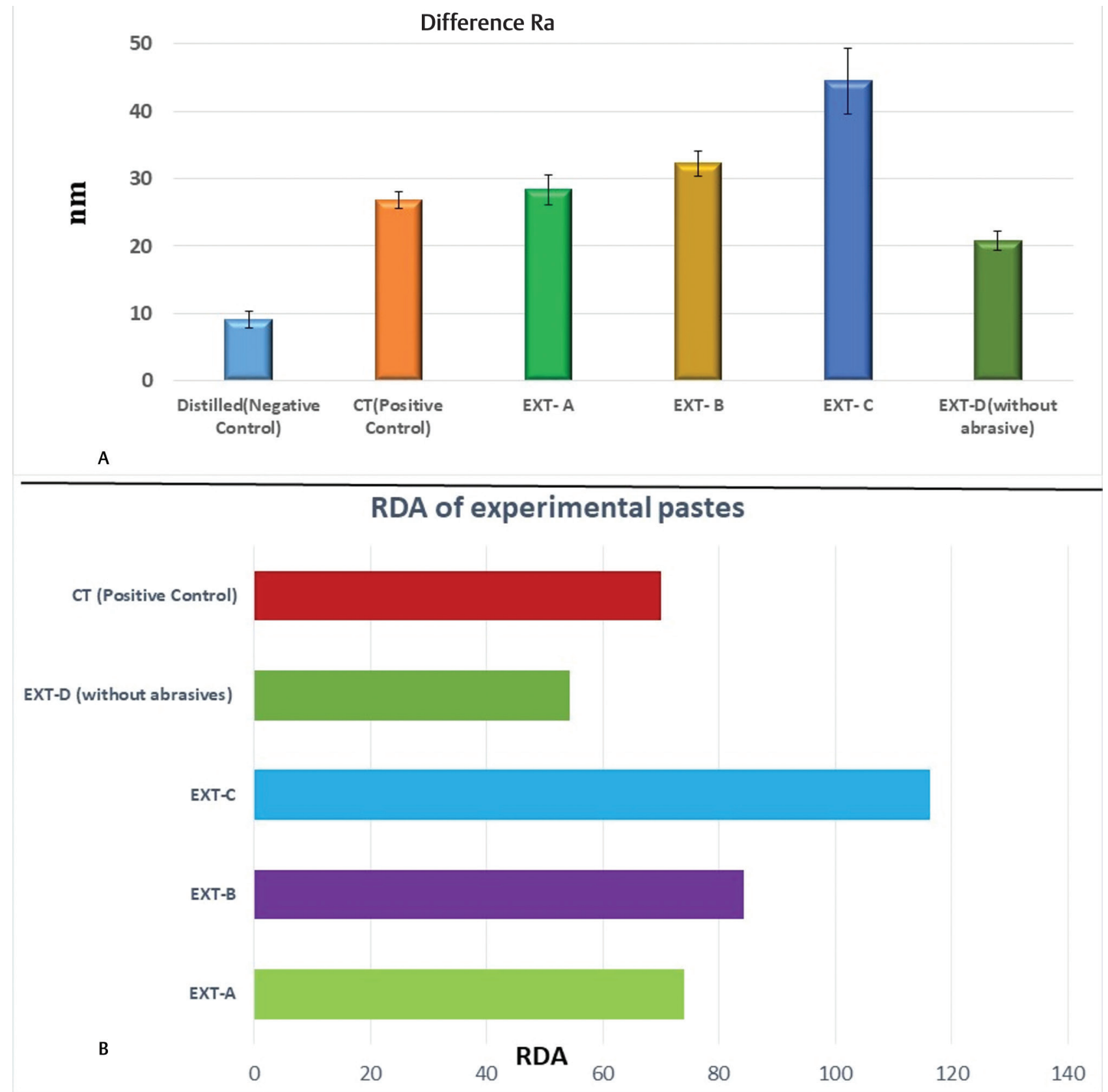

Fig. 1 (A) Mean plot of difference-roughness average (Ra) with 95\% confidence interval, and (B) relative dentin abrasivity (RDA) of experimental and control dentifrices (CT).

Tamhane's tests were used for multiple comparisons. The level of significance was set at $p<0.05$.

\section{Results}

\section{AFM Examination}

The results of the present study demonstrated significant differences in the abrasion values of the various investigated groups ( $p<0.05$ ) except CT and EXT-A, where there was nonsignificant difference between these two groups $(p=0.76$ and $p=0.80$, respectively). The graphic representation of $\mathrm{Ra}$ and RDA is given in - Fig. 1A, B respectively. The surface of all groups showed signs of abrasion even when the brush was used with distilled water. The 3D images of abraded and nonabraded dentins are illustrated in - Fig. 2A-G. The untreated portion (polished surface) presented relatively low values ( $6.11 \pm 0.34 \mathrm{~nm}$ ) of surface roughness. However, as expected, toothbrush abrasion caused visible nano-scale alterations on the surface of all samples, varying in extent, according to the toothpaste employed as illustrated by $3 \mathrm{D}$ images obtained with the help of AFM shown in - Fig. 2A-G. The negative $\mathrm{CT}$ group (distilled water) showed the least surface roughness $(\mathrm{Ra}=9.08 \pm 1.03 \mathrm{~nm}$ ), whereas EXT-C group showed the highest surface roughness $(\mathrm{Ra}=44.53 \pm 3.93 \mathrm{~nm})$ compared to $\mathrm{CT}(\mathrm{Ra}=26.8 \pm 1.05 \mathrm{~nm})$. EXT-A and EXT-B groups showed slightly more surface roughness $(\mathrm{Ra}=28.36 \pm 1.77$ $\mathrm{nm}$ and $32.28 \pm 1.48 \mathrm{~nm}$, respectively) when compared with a positive CT group, whereas EXT-D group (without abrasives) 

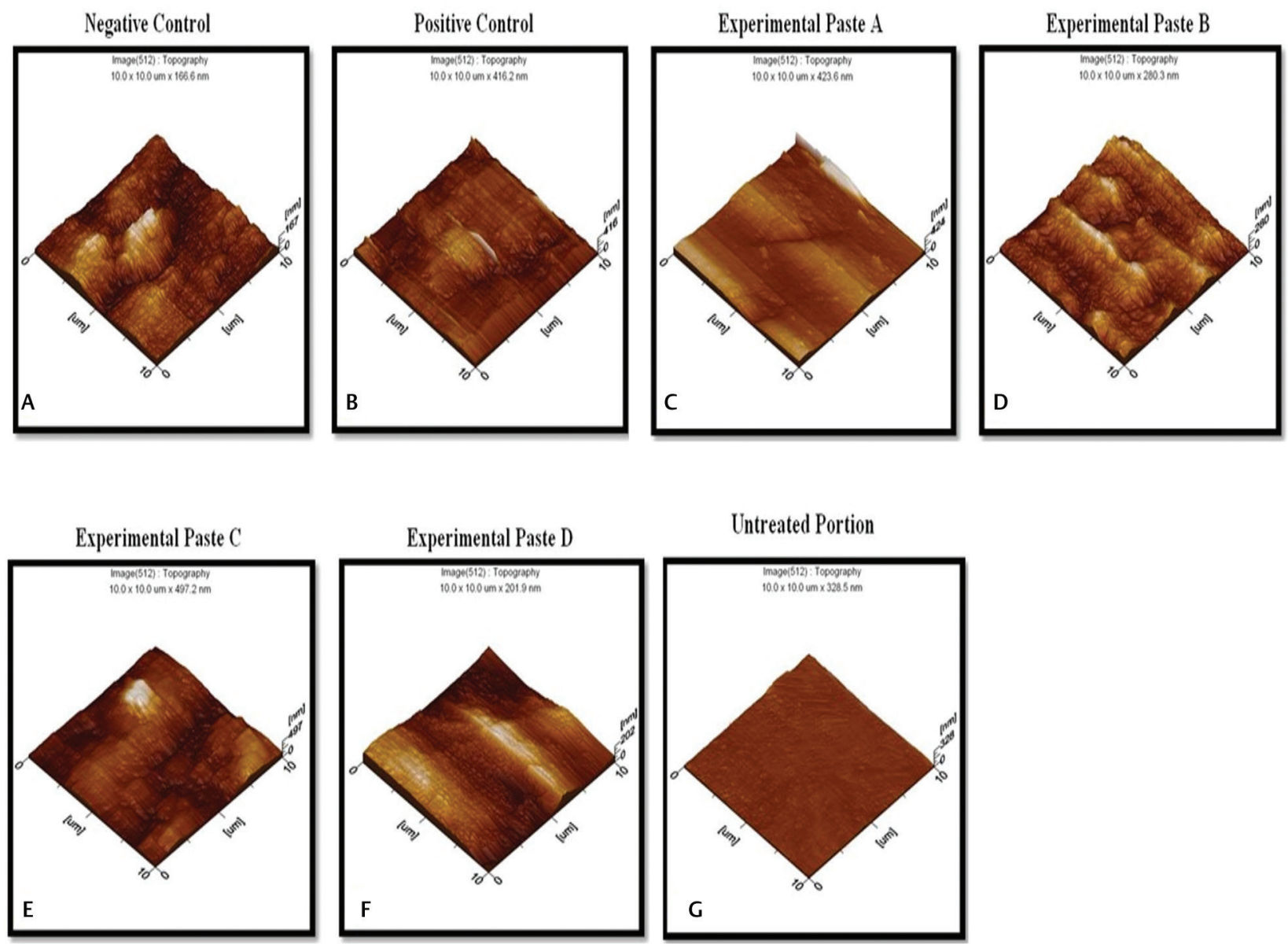

Fig. 2 Comparison of three-dimensional (3D) images of surface topography of different groups by atomic force microscopy (AFM): (A) distilled water, (B) control dentifrice (CT), (C) EXT-A, (D) EXT-B, (E) EXT-C, (F) EXT-D, and (G) untreated portion.

produced lesser surface roughness $(\mathrm{Ra}=20.76 \pm 1.16 \mathrm{~nm})$ than CT (positive CT group).

\section{SEM-EDX Examination}

SEM micrographs of etched dentin presented with open or patent dentinal tubules are given in - Fig. 3A. For samples, which were rinsed with distilled water followed by sonication for 30 seconds, treatment with distilled water showed (-Fig. 3B) smooth dentin surface with open or patent dentinal tubules. CT group showed $(\boldsymbol{-}$ Fig. $\mathbf{3 C}$ ) relatively smooth dentin surface with open dentinal tubules and few particles on the dentin surface and some were partially obliterated, whereas EXT-A, EXT-B, and EXT-C groups showed ( - Fig. 3D-F) a smear layer-like coating covering all the dentin surface and uniform occlusion of almost all the dentinal tubules. Very few tubule orifices remained partially opened with maximum narrowing of the tubular lumen. The EXT-D group showed ( - Fig. 3G) relatively smooth surface with open dentinal tubules with the presence of few particles on the dentin surface and some were partially obliterated. The mean \pm SD percentage of tubule occlusion accessed by independent reviewers were $12.42 \pm 1.74 \%, 99.16 \pm 1.88 \%, 99.80$ $\pm 0.45 \%, 99.82 \pm 0.40 \%$, and $6.66 \pm 1.48 \%$ for CT, EXT-A, EXT-B,
EXT-C, and EXT-D, respectively. Post-hoc analysis indicated that EXT-A, EXT-B, and EXT-C performed equally and there was an insignificant difference $(p>0.05)$ in terms of tubule occlusion competence, whereas CT and EXT-D were similar in performance.

SEM micrographs of samples, which were rinsed only after brushing simulation, produced similar results in terms of tubule occlusion competence. Before sonication, the samples showed ( - Fig. $4 \mathbf{A A}-\mathbf{F}$ ) the presence of many particles, whereas after sonication loose particles were washed away; however, the dentinal tubules remain occluded for all the samples treated with novel dentifrices.

EDX analysis commenced on the etched dentin surface showed (Fig not shown) peaks of carbon and oxygen, while calcium, phosphate, and sodium peaks were not observed as major elements. The samples treated with distilled water, CT, and EXT-D showed calcium, silicon, and sodium peaks with low intensity. However, EXT-A, EXT-B, and EXT-C showed dominant peaks of calcium and phosphate along with the presence of silicon, sodium, fluoride, and zinc as shown in -Fig. 5A-C, whereby the obtained $\mathrm{Ca} / \mathrm{P}$ of these treated samples were $1.93,1.73$, and 2.15 , respectively. The particle size analysis of samples treated with EXT-A, EXT-B, and EXT-C 

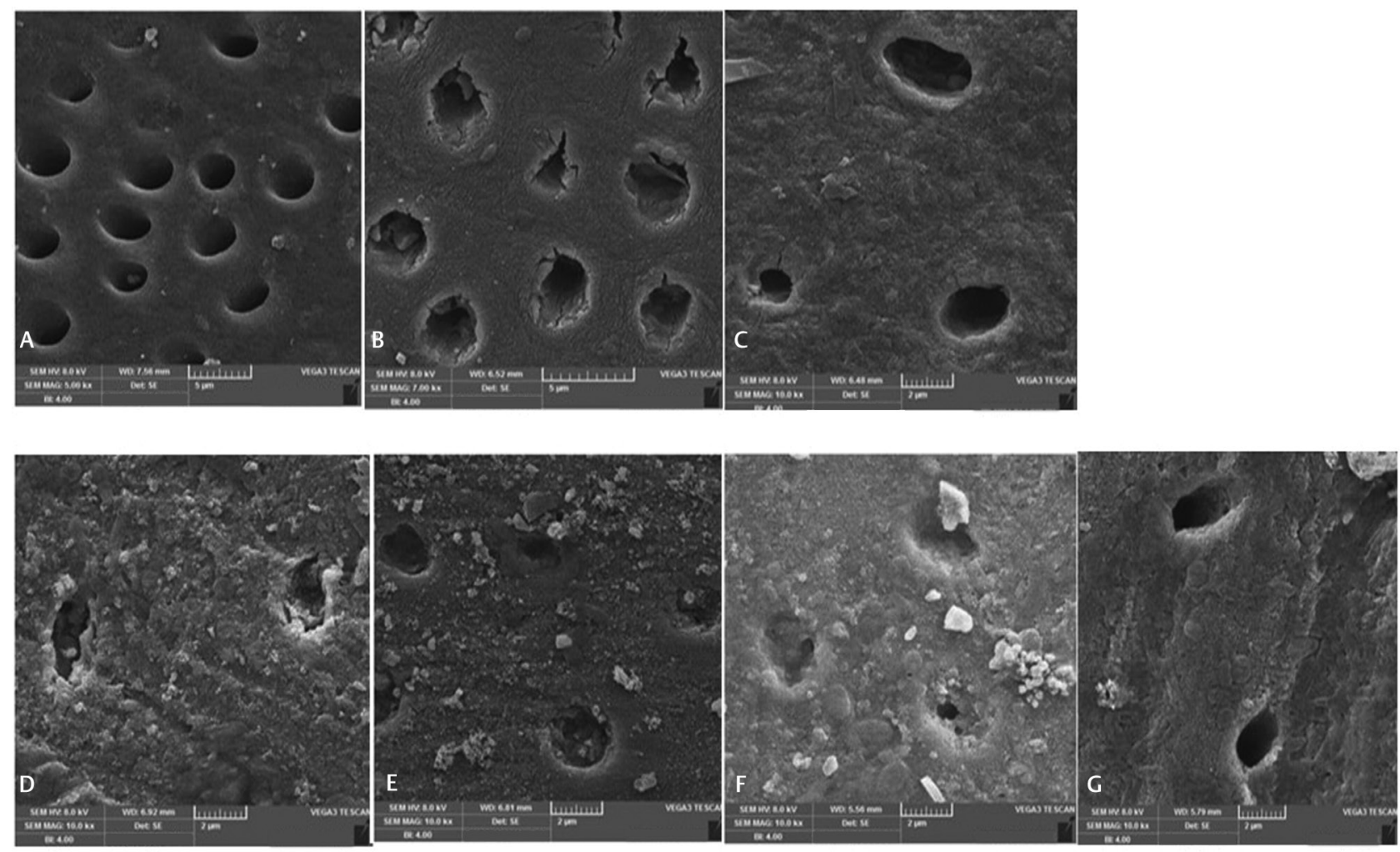

Fig. 3 (A) Scanning electron microscopy (SEM) micrograph of dentin surface morphology etched with 37\% phosphoric acid showing open or patent dentinal tubules, (B-G) SEM micrographs of all six groups at magnifications $\times 10,000$ after rinsing and sonication, (B) distilled water, (C) control dentifrice (CT), (D) EXT-A, (E) EXT-B, (F) EXT-C, and (G) EXT-D.

showed (Supplementary Fig. S1 [online only]) attachment of nano-sized n-FBG and n-ZnO on the dentinal surface and occluded almost all the dentinal tubules.

\section{Discussion}

The obtained results of EXT dentifrices with respect to abrasivity are within the acceptable range. The acceptable limit value of RDA set by the American Dental Association (ADA) and the Food and Drug Authority (FDA) is 250 and 200, respectively (ISO-11609:2010). The maximum RDA value was obtained with EXT-C, that is, 116.24, where the concentration of n-FBG was $3.5 \mathrm{wt} . \%$ in addition to $\mathrm{n}-\mathrm{ZnO}$ (3 wt.\%) and thymoquinone (3 wt.\%). The RDA value of EXT-A (74.04; n-FBG 1.5 wt.\%) was almost similar to $\mathrm{CT}$ (70). This difference in the abrasivity values might be due to different concentrations of n-FBG used for the EXT pastes A, B, and C. With the increase in the concentration of n-FBG, the abrasivity increased. It is reported that fluoridated bioactive glasses with low glass transition temperature and a more disrupted glass network are softer and are, therefore, less abrasive toward enamel. ${ }^{23}$ The abrasivity of dentifrices is dependent on numerous factors such as hardness, shape, size, and concentration of its abrasive components. ${ }^{17,24}$ It is reported that the abrasive wear rate increases linearly as the particle size and concentration is increased to a critical size. ${ }^{25}$ Typically, the particle size and shape of the abrasive particles in the range of $1-20 \mu \mathrm{m}$ is desirable to provide cleaning without damaging hard tissues and should not be sharp or angular. ${ }^{26}$ The SEM images of this present study showed that the particle shape of n-FBG was spherical and nano-rod-shaped $\mathrm{ZnO}$, where the size of the abrasive particles used was at the nano-scale $(80-250 \mathrm{~nm})$. Therefore, it is likely that the abrasive particles used for the EXT dentifrices are too small to be abrasive and the nature of the abrasive particles maintained the level of abrasiveness.

This study also found that EXT-D (without abrasives) produced significantly lesser abrasion $(\mathrm{Ra}=20.76 \mathrm{~nm})$ and a lower RDA value of 54.20 compared with 70 . This significantly lesser abrasion produced was likely, as EXT paste D did not contain any abrasive agents. It is pertinent to mention that other ingredients such as calcium carbonate $\left(\mathrm{CaCO}_{3}\right)$ added as a basic ingredient in the EXT formulations $\mathrm{A}, \mathrm{B}, \mathrm{C}$, and D might also contribute to the abrasivity of toothpastes as $\mathrm{CaCO}_{3}$ is one of the most commonly used abrasives in toothpastes. All toothpastes contain a variety of ingredients, which makes it almost impossible to determine the influence of a certain substance on the abrasivity of toothpaste. ${ }^{27}$

In the present study after the brushing experiments, the SEM images depicting the dentinal tubules of initially demineralized (etched) samples were completely open. Samples treated with distilled water and CT also showed open or patent dentinal tubules with few particles on the dentin surface of Colgate-treated samples and some partially obliterated. As expected, the samples treated with EXT-D without any active ingredients presented with open tubules and few particles on the dentin surface. In contrast, all the samples treated with EXT dentifrices not only created a smear 

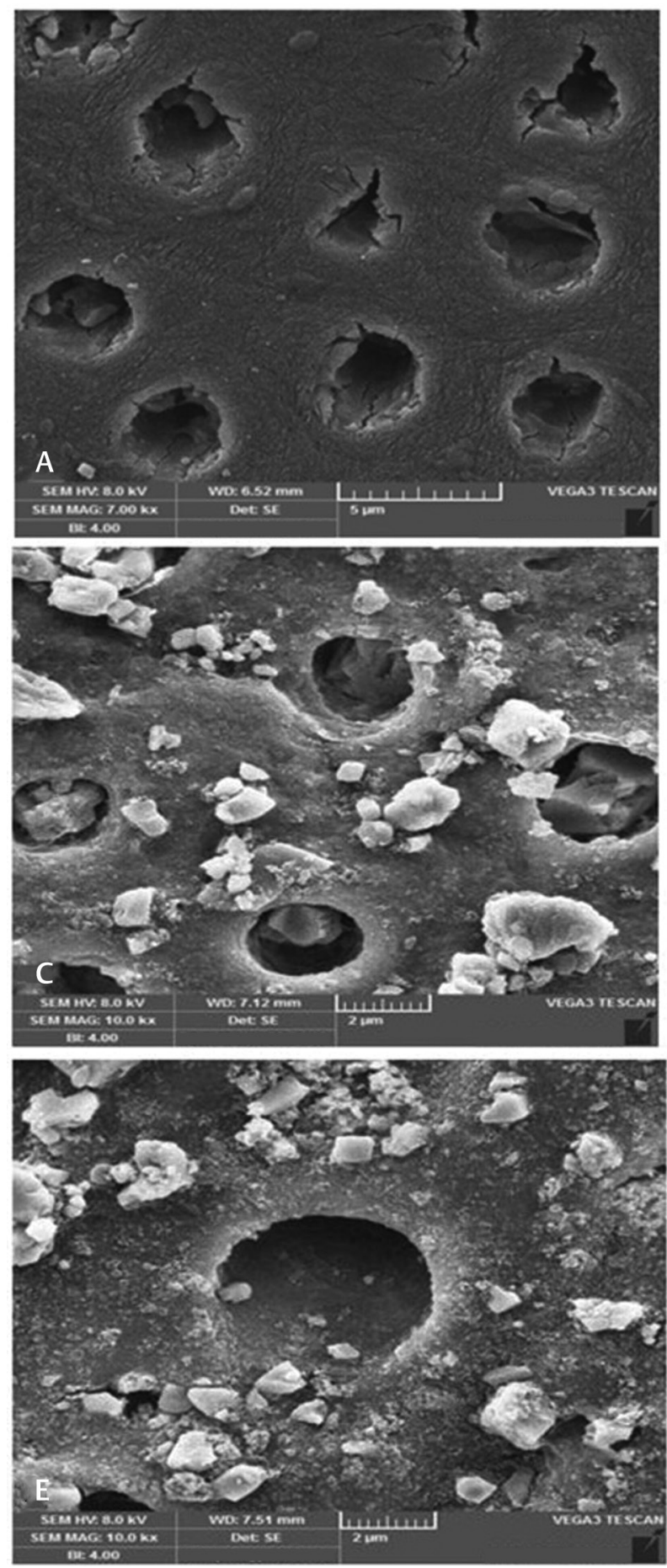
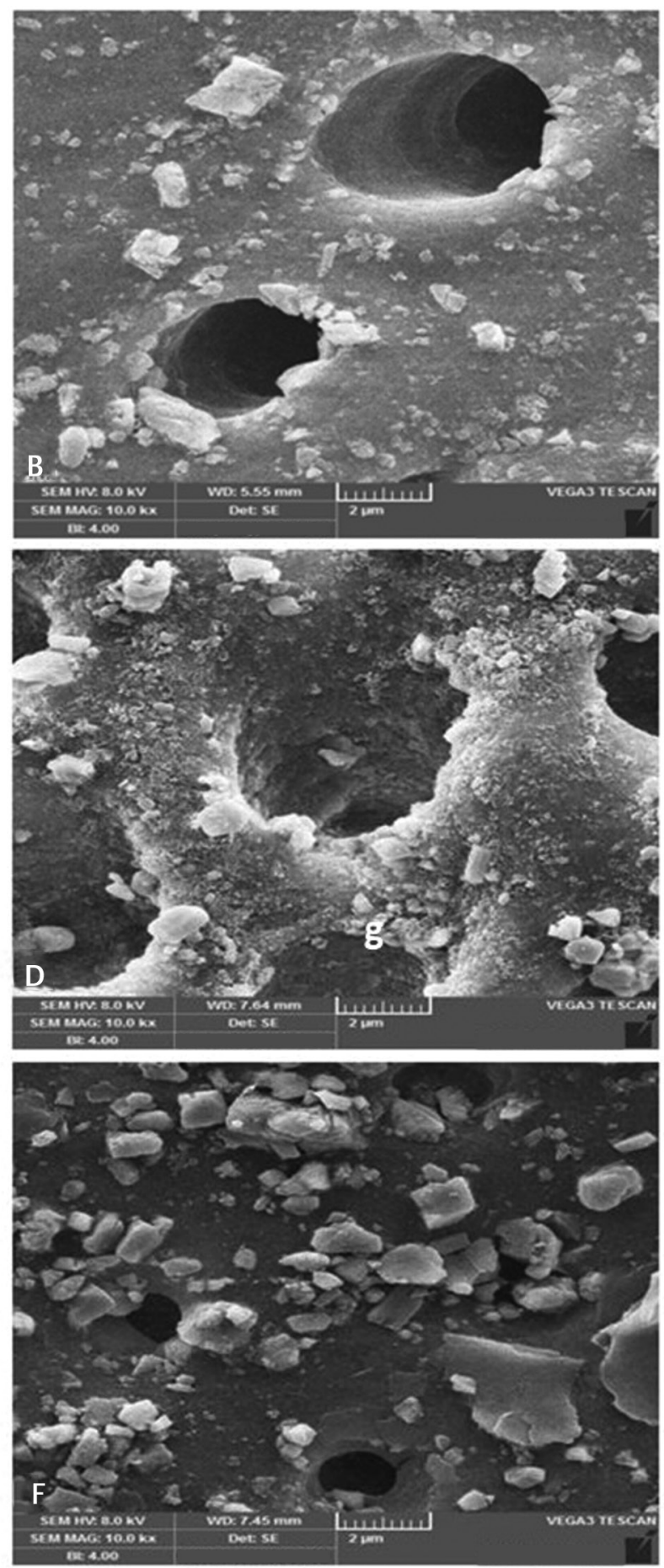

Fig. 4 Scanning electron microscopy (SEM) micrographs of all six groups at magnifications $\times 10,000$ after rinsing with distilled water only. (A) Distilled water, (B) control dentifrice (CT), (C) EXT-A, (D) EXT-B, (E) EXT-C, and (F) EXT-D.

layer-like coating on the dentin surface but also uniformly occluded almost all the dentinal tubules. Very few tubules remained partially open with maximum narrowing of the tubular lumen. This suggested that three different concentrations of n-FBG used for EXT groups were equally effective in occluding open dentinal tubules. These results of the present study are in accordance with the findings of a previous study. ${ }^{22}$ Thus, all n-FBG treated samples examined in this study exhibited material deposition and successful occlusion of dentinal tubules, which would relieve DH.

It is pertinent to mention that SEM images of samples rinsed with distilled water only after brushing simulation and samples rinsed with distilled water and sonicated for the 30 seconds presented similar results in terms of tubule occlusion competence. Before sonication, the samples showed presence of many particles on dentin surface, whereas 

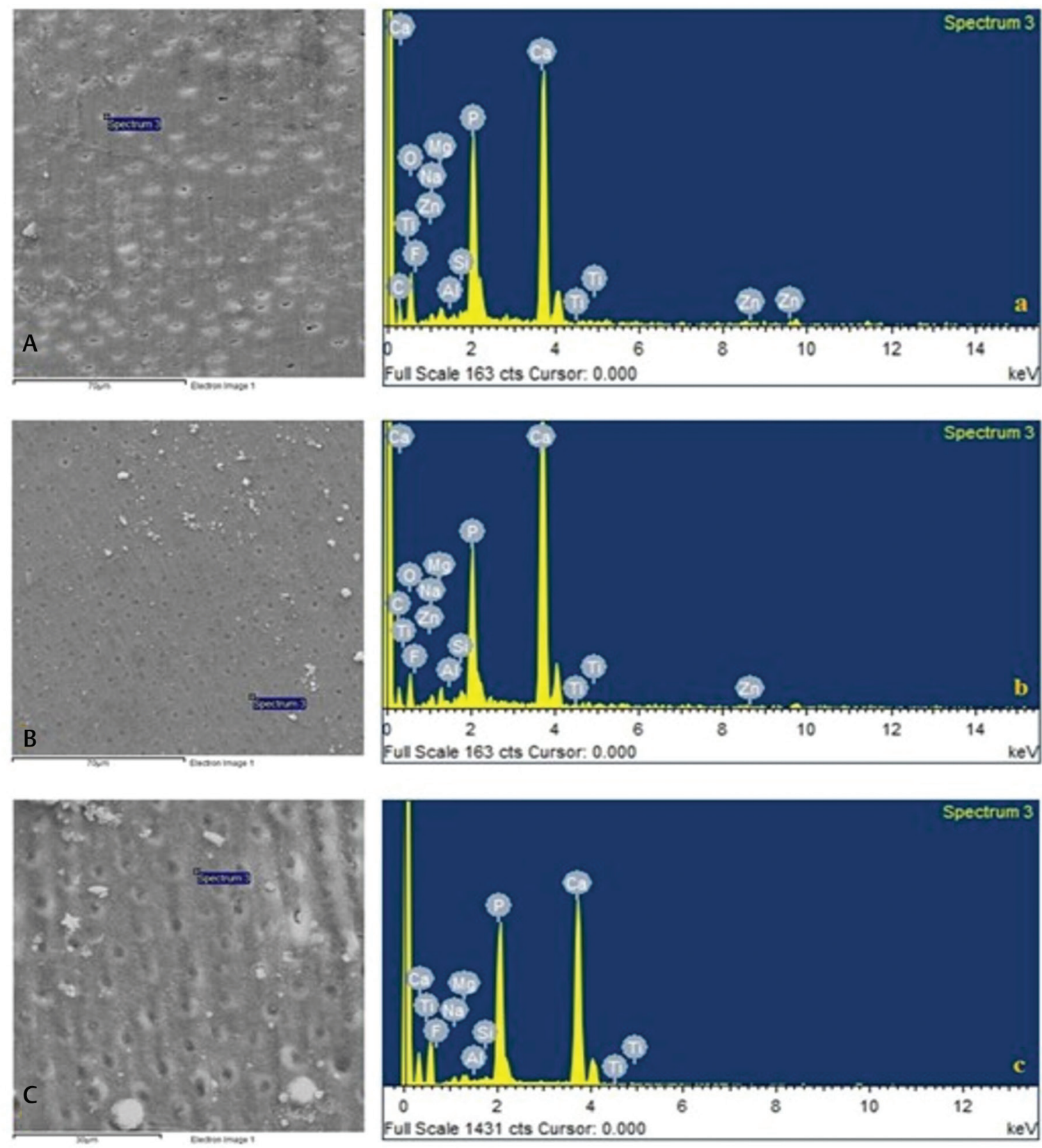

Fig. 5 Energy dispersive X-ray (EDX) of samples treated with nano-sized fluoride-containing bioactive particles (n-FBG) and nano-oxides $(\mathrm{n}-\mathrm{ZnO})$ showing point scan elemental analysis and attachment selection of particles attached to the samples in groups with experimental toothpaste slurries: (A) EXT-A, (B) EXT-B, and (C) EXT-C.

after sonication loose particles were washed away; however, the dentinal tubules remained occluded for all the samples treated with novel dentifrices A, B, and C. These results showed good retentive properties of these EXT dentifrices as sonication after brushing experiments did not produce any noticeable difference in terms of tubule occlusion competence. It suggests that the novel dentifrices can withstand dynamic oral conditions. In the present study, this mineral deposition was confirmed by the results of EDX. The Ca/P ratios of the dentin surface depositions were higher than that of natural hydroxyapatite $(\mathrm{Ca} / p=1.67)$, which might be explained by the residual BG on the dentin surfaces. Hence, the novel dentifrices $\mathrm{A}, \mathrm{B}$, and $\mathrm{C}$ formulated in this study have the ability to get adsorbed on the dentin providing the mineral content. It was confirmed from previous studies that BG could promote mineral formation on dentin surfaces. ${ }^{28}$ The toothpaste matrix is likely to encapsulate BG particles and slow the dissolution/precipitation mechanism. This delay in bioactivity could be attributed to a change in ionic mobility due to a large number of organics present in toothpastes and the possible interaction between the different ions in solution and surfactant that compose the dentifrice. ${ }^{29}$ It could be expected as well that fluoride release from toothpastes containing F-BGs would be less as compared with the BG alone 
due to the likeliness of encapsulation of F-BGs in toothpaste matrix. The n-FBG particles can be lodged in the dentinal tubules due to their smaller size by mechanical brushing, enabling the dissolution of the glass. The tubular structure then determines the calcium $\left(\mathrm{Ca}^{+}\right)$, phosphorus $\left(\mathrm{PO}_{4}\right)$, and hydroxyl $\left(\mathrm{OH}^{-}\right)$ion deposition and subsequent apatite propagation and occlusion to occur. Also, n-FBG particles used in this study had a larger surface area to volume ratio $\left(64.3 \mathrm{~m}^{2} \cdot \mathrm{g}^{-1}\right)$, which may further increase the area of glass network exposed to dissolution resulting in increased dissolution rate. ${ }^{6,20}$

The present study used $\mathrm{n}$ - $\mathrm{ZnO}$ added as an active ingredient in the EXT formulation, where the zinc has bactericidal, anticaries, and antigingivitis actions. ${ }^{30,31}$ The EDX spectra of the samples after brushing showed the presence of $\mathrm{Zn}^{2+}$, which confirmed the mineral deposition by the active ingredient $(\mathrm{n}-\mathrm{ZnO})$. Point scan elemental analysis further confirmed the presence of $\mathrm{Zn}^{2+}$ in the occluding material of dentinal tubules. Ideally, a sustained release of ions (zinc and fluoride) from toothpaste formulations is recommended to deliver a therapeutic effect in vivo. Hence, the zinc and F-BGs can act as effective desensitizers into the novel remineralizing toothpastes $\mathrm{A}, \mathrm{B}$, and $\mathrm{C}$.

Thymoquinone has also been added as an active ingredient in the novel dentifrices. It is anticipated that the addition of thymoquinone in the novel dentifrices could provide additional benefits such as to restrict the abrasivity of dentin. The polymeric structure would plasticize under the mechanical force during toothbrushing. Therefore, it is expected that the black seed essential oil and its active compound, thymoquinone, will leach out, which would not only perform antibacterial and anti-inflammatory responses but would also flow in dentinal tubules and help in reducing $\mathrm{DH}$ and abrasivity.

With the present methodology, the occluding/remineralization potential of $\mathrm{n}$-FBG and $\mathrm{n}$ - $\mathrm{ZnO}$ was determined effectively by using a CT group and replicating the clinical procedures in vitro. The positive results obtained from this study showed substantial tubule occlusion, and release of therapeutically active ions such as fluoride and zinc are encouraging and present valuable data to be used for other $\mathrm{n}-\mathrm{FBG}$ and $\mathrm{n}-\mathrm{ZnO}$ containing dentifrices, as it will provide a sound premise for future studies.

\section{Conclusion}

All novel n-FBG dentifrices fall within the acceptable range of RDA. With the increase in the concentration of n-FBG, the abrasivity increased; however, the values were within the acceptable range set by international standards. Regarding occluding potential, all three novel dentifrices A, B, and C presented similar results in terms of tubule occlusion competence. Among all the study groups, EXT-A was found to be the best as it not only exhibited the lowest surface roughness and RDA value but also showed nearly the highest tubule occlusion. Therefore, it may be recommended for treating $\mathrm{DH}$ for daily use in individuals with a compromised periodontal status and individuals with a compromised enamel surface. This novel dentifrice would provide a mild abrasive action for the removal of stains and at the same time would reinforce the tooth structure by remineralization and provide relief from $\mathrm{DH}$.

It is recommended that for EXT-B and EXT-C with obtained $\mathrm{RDA}$ value may be recommended as a whitening toothpaste for use in individuals with extrinsic staining, which may be caused by smoking, pigments in beverages and foods, for the removal of stubborn, hard, and tougher stains. This novel dentifrice would have a combined effect of physically removing the tough stains due to stronger abrasive action and also providing relief from $\mathrm{DH}$ at the same time, which is a unique combination contrary to the risks of tooth whitening such as increased tooth sensitivity and gingival irritation.

\section{Conflict of Interest}

None declared.

\section{Acknowledgments}

The authors would like to acknowledge Engr. Asim (IRCBM, COMSATS University Islamabad, Lahore Campus, Lahore, Pakistan) for helping in SEM analysis and Dr. Peter John (National University of Sciences and Technology, Islamabad, Pakistan) for helping in AFM characterization.

\section{References}

1 Jena A, Kala S, Shashirekha G. Comparing the effectiveness of four desensitizing toothpastes on dentinal tubule occlusion: a scanning electron microscope analysis. J Conserv Dent 2017;20(4):269-272

2 Wang Z, Jiang T, Sauro S, et al. The dentine remineralization activity of a desensitizing bioactive glass-containing toothpaste: an in vitro study. Aust Dent J 2011;56(4):372-381

3 Idon P, Esan T, Bamise C, et al. Dentine hypersensitivity: review of a common oral health problem. J Dent Craniofac Res 2017;2(2):16

4 Miglani S, Aggarwal V, Ahuja B. Dentin hypersensitivity: recent trends in management. J Conserv Dent 2010;13(4):218-224

5 AlSubaie AA, Sarfraz Z, AlAli AA, et al. Effect of nano-zinc oxide and fluoride-doped bioactive glass-based dentifrices on esthetic restorations. Dent Med Probl 2019;56(1):59-65

6 Curtis AR, West NX, Su B. Synthesis of nanobioglass and formation of apatite rods to occlude exposed dentine tubules and eliminate hypersensitivity. Acta Biomater 2010;6(9):3740-3746

7 Madhurkar JG, Bhat PR, Acharya AB, Thakur SL, Trasad VA. Efficacy of milk as a desensitizing agent for the treatment of sensitivity following scaling and root planing. Contemp Clin Dent 2017;8(2):231-235

8 Gillam DG, Tang JY, Mordan NJ, Newman HN. The effects of a novel bioglass dentifrice on dentine sensitivity: a scanning electron microscopy investigation. J Oral Rehabil 2002;29(4):305-313

9 Shivani S, Tumkur SA, Onkar K, Chetan P, Namrata H. Efficacy of novamin- and pro-argin-containing desensitizing dentifrices on occlusion of dentinal tubules. Dent Hypotheses 2017;8(4):104-109

10 Brauer DS, Karpukhina N, O'Donnell MD, Law RV, Hill RG. Fluoride-containing bioactive glasses: effect of glass design and structure on degradation, $\mathrm{pH}$ and apatite formation in simulated body fluid. Acta Biomater 2010;6(8):3275-3282 
11 De Caluwé T, Vercruysse CWJ, Declercq HA, Schaubroeck D, Verbeeck RM, Martens LC. Bioactivity and biocompatibility of two fluoride containing bioactive glasses for dental applications. Dent Mater 2016;32(11):1414-1428

12 Lynch E, Brauer DS, Karpukhina N, Gillam DG, Hill RG. Multi-component bioactive glasses of varying fluoride content for treating dentin hypersensitivity. Dent Mater 2012;28(2):168-178

13 Al-Bayaty F, Kamaruddin AA, Ismail M, Abdulla M. Formulation and evaluation of a new biodegradable periodontal chip containing thymoquinone in a chitosan base for the management of chronic periodontitis. J Nanomater 2013; (3):5

14 Ghelichli M. Formulation of new herbal Salvadora toothpaste with antidecay, wound healing and caring tooth and periodontal diseases. Bull Environ Pharmacol Life Sci 2014;3(11):115-122

15 Jalaluddin M, Rajasekaran UB, Paul S, Dhanya RS, Sudeep CB, Adarsh VJ. Comparative evaluation of neem mouthwash on plaque and gingivitis: a double-blind crossover study. J Contemp Dent Pract 2017;18(7):567-571

16 Al-Attass SA, Zahran FM, Turkistany SA. Nigella sativa and its active constituent thymoquinone in oral health. Saudi Med J 2016;37(3):235-244

17 Lippert F. An introduction to toothpaste - its purpose, history and ingredients. Monogr Oral Sci 2013;23:1-14

18 International Organization for Standardization 11609. Dentistry - Dentifrices - Requirements, test methods and marking. Geneva: International Organization for Standardization; 2010:20

19 Ahtzaz S, Nasir M, Shahzadi L, et al. A study of the effect of zinc oxide and zinc peroxide nanoparticles to enhance angiogenesis-pro-angiogenic grafts for tissue regeneration applications. Mater Des 2017;132:409-418

20 Gul H, Zahid S, Zahid S, et al. Sol-gel derived fluoride-doped bioactive glass powders: structural and long-term fluoride release/pH analysis. J Non-Cryst Solids 2018;498:216-222

21 Mahmood A, Mneimne M, Zou LF, Hill RG, Gillam DG. Abrasive wear of enamel by bioactive glass-based toothpastes. Am J Dent 2014;27(5):263-267
22 Farooq I, Moheet IA, AlShwaimi E. In vitro dentin tubule occlusion and remineralization competence of various toothpastes. Arch Oral Biol 2015;60(9):1246-1253

23 Chen X, Shah P, Mneimne M, Hill R, Karpukhina N. The retarding effect of zinc oxide on dissolution and apatite formation of a fluoride containing bioactive glass. Paper presented at: European Conference on Biomaterials; August 31-September 04, 2014; Liverpool, UK

24 Hara AT, Turssi CP. Baking soda as an abrasive in toothpastes: mechanism of action and safety and effectiveness considerations. J Am Dent Assoc 2017;148(11S) :S27-S33

25 Ganss C, Möllers M, Schlueter N. Do abrasives play a role in toothpaste efficacy against erosion/abrasion? Caries Res 2017;51(1):52-57

26 Singh RP, Sharma S, Logani A, Shah N, Singh S. Comparative evaluation of tooth substance loss and its correlation with the abrasivity and chemical composition of different dentifrices. Indian J Dent Res 2016;27(6):630-636

27 Arnold WH, Gröger Ch, Bizhang M, Naumova EA. Dentin abrasivity of various desensitizing toothpastes. Head Face Med 2016;12(1):16

28 Sheng XY, Gong WY, Hu Q, Chen Xf, Dong YM. Mineral formation on dentin induced by nano-bioactive glass. Chin Chem Lett 2016;27(9):1509-1514

29 Maçon ALB, Valliant ES, Earl J, Jones J. Bioactivity of toothpaste containing bioactive glass in remineralizing media: effect of fluoride release from the enzymatic cleavage of monofluorophosphate. Biomed Glasses 2015;1(1):41-50

30 Cummins D. Zinc citrate/Triclosan: a new anti-plaque system for the control of plaque and the prevention of gingivitis: short-term clinical and mode of action studies. J Clin Periodontol 1991;18(6):455-461

31 Kasraei S, Sami L, Hendi S, Alikhani MY, Rezaei-Soufi L, Khamverdi Z. Antibacterial properties of composite resins incorporating silver and zinc oxide nanoparticles on Streptococcus mutans and Lactobacillus. Restor Dent Endod 2014;39(2):109-114 